\title{
A philicity based analysis of adsorption of small molecules in zeolites
}

\author{
ANGELES CUÁN ${ }^{1,2}$, MARCELO GALVÁN ${ }^{1,3, *}$ and PRATIM KUMAR CHATTARAJ ${ }^{1,4, *}$ \\ ${ }^{1}$ Departamento de Química, División de Ciencias Básicas e Ingeniería, Universidad Autónoma \\ Metropolitana-Iztapalapa, AP 55-534, México DF, 09340, México \\ ${ }^{2}$ Present address: Programa de Ingeniería Molecular, Instituto Mexicano del Petróleo, Eje Lázaro \\ Cárdenas 152, México DF, 07730, México \\ ${ }^{3}$ Theory of Condensed Matter Group, Cavendish Laboratory, University of Cambridge, Cambridge \\ CB3 0HE, UK \\ ${ }^{4}$ Department of Chemistry, Indian Institute of Technology, Kharagpur 721302, India \\ e-mail: mgalvan@xanum.uam.mx; pkc@chem.iitkgp.ernet.in
}

\begin{abstract}
Adsorption of small molecules like $\mathrm{CH}_{4}, \mathrm{CO}$ and $\mathrm{NH}_{3}$ into the acid sites of zeolites is analysed as an interaction between an electrophile and a nucleophile. Global reactivity descriptors like softness and electrophilicity, and local reactivity descriptors like the Fukui function, local softness and local philicity are calculated within density functional as well as Hartree-Fock frameworks using both Mulliken and Hirshfeld population analysis schemes. The HSAB principle and the best electrophilenucleophile combination suggest that the reaction between the $\mathrm{NH}_{3}$ and Brönsted acid site of the zeolite is the strongest. Interaction between the zeolite and a small probe molecule takes place through the most electrophilic atom of one with the most nucleophilic atom of the other. This result is in conformity with those provided by the frontier orbital theory and the local HSAB principle.
\end{abstract}

Keywords. Density functional theory; Hartree-Fock calculations; Global and local reactivity descriptors; HSAB principle; electrophile; nucleophile.

\section{Introduction}

There has been an upsurge of interest in recent years in understanding the catalysis phenomenon using zeolites. Most of these studies ${ }^{1-8}$ highlight the creation of Brönsted acid sites owing to the substitution of silicon by aluminum in the tetrahedral units of the zeolite framework. It is important to know the variation of reactivity and selectivity in those acid sites when small molecules like $\mathrm{CH}_{4}, \mathrm{CO}$ and $\mathrm{NH}_{3}$ get adsorbed there, in order to have a better understanding of the associated catalytic activity. Recently, Deka et $a l^{8}$ have studied this aspect using local softness.

Density functional theory ${ }^{9,10}$ (DFT) has been found to be quite successful in analysing chemical reactivity and selectivity. While global quantities like electronegativity ${ }^{11,12}(\chi)$, hardness ${ }^{13-15}(\eta)$, and electrophilicity index ${ }^{16}(\omega)$ take care of the reactivity, the selectivity of each atomic site in a molecule is characterized by local quantities like the Fukui function ${ }^{17,18}(f(\mathbf{r}))$, local softness ${ }^{19}(s(\mathbf{r}))$ and philicity ${ }^{20}(\omega(\mathbf{r}))$.

Various electronic structure principles involving the global reactivity descriptors exist. The important one for the present study is the hard-soft acid-

\footnotetext{
*For correspondence
}

base $^{13,21}$ (HSAB) principle which states that "hard acids like to coordinate with hard bases and soft acids with soft bases for both their kinetic and thermodynamic properties". A local version of this principle is also available ${ }^{22}$ through the matching of local softness values. An electrophile-nucleophile interaction can become favorable between two molecules with high and low electrophilicity indices respectively so that the former is a better electrophile and the latter may be considered as a better nucleophile. ${ }^{16}$ Corresponding philicities ${ }^{20}$ may be used in analysing the specific site-site interactions.

In the present work, we study the nature of adsorption of small molecules like $\mathrm{CH}_{4}, \mathrm{CO}$ and $\mathrm{NH}_{3}$ inside the zeolite framework especially into the Broensted acidic hydrogen atomic sites. We use the global electrophilicity and local philicity for this purpose. Theoretical background is provided in $\S 2$ and in $\S 3$ we present the computational details. Results are discussed in $\S 4$ and $\S 5$ contains some concluding remarks.

\section{Theoretical background}

Electronegativity ${ }^{11}(\chi)$, and hardness ${ }^{13}(\eta)$, for an $N$ electron system with total energy $E$, are defined as follows: 


$$
\begin{aligned}
& \chi=-\mu=-(\partial E / \partial N)_{v(\mathbf{r}),} \\
& \eta=(1 / 2)\left(\partial^{2} E / \partial N^{2}\right)_{\mathrm{v}(\mathbf{r})}=(1 / 2)(\partial \mu / \partial N)_{v(\mathbf{r})}
\end{aligned}
$$

where $\mu$ and $\mathrm{V}(\mathbf{r})$ are chemical and external potentials, respectively. The global softness $(S)$ is the following inverse of hardness, ${ }^{9}$

$$
S=1 / 2 \eta=(\partial N / \partial \mu)_{v(\mathbf{r})}
$$

The elecrophilicity index $(\omega)$ is introduced by Parr et al $^{16}$ as follows:

$$
\omega=\mu^{2} / 2 \eta
$$

Local quantities are important in gaining insights into the reactivity and selectivity of a specific site in a molecule.

The Fukui function ${ }^{17}$ or the frontier function captures the essence of Fukui's frontier orbital theory ${ }^{23}$ and is defined as

$$
f(\mathbf{r})=(\partial \rho(\mathbf{r}) / \partial N)_{\mathrm{v}(\mathbf{r})}=(\delta \mu / \delta \mathrm{v}(\mathbf{r}))_{N}
$$

Owing to the discontinuities in the plot of $\rho(\mathbf{r})$ versus $N$ there exist three different types of Fukui functions, ${ }^{17}$ namely,

$f^{+}(\mathbf{r})=\rho_{N+1}(\mathbf{r})-\rho_{N}(\mathbf{r})$, for nucleophilic attack,

$f^{-}(\mathbf{r})=\rho_{N}(\mathbf{r})-\rho_{N-1}(\mathbf{r}), \quad$ for electrophilic attack,

$f^{0}(\mathbf{r})=\rho_{N+1}(\mathbf{r})-\rho_{N-1}(\mathbf{r}) / 2, \quad$ for radical attack,

where $f_{N}(\mathbf{r})$ is the electron density of the $N$-electron $(N \equiv N-1, N, N+1)$ species. Substituting these electron densities by respective electron populations $\left(q_{k}\right)$ on the atomic site $k$ of the molecule one can define the corresponding condensed-to-atom variants as,

$$
f_{k}^{\alpha} ; \alpha=+,-, \text { and, } 0
$$

where $\alpha=+,-$, and 0 represent nucleophilic, electrophilic, and radical attacks respectively.

The local softness is given by ${ }^{9}$

$$
\begin{aligned}
s(\mathbf{r})= & (\partial \rho(\mathbf{r}) / \partial \mu)_{\vee(\mathbf{r})}=(\partial \rho(\mathbf{r}) / \partial N)_{\vee(\mathbf{r})} \\
& (\partial N / \partial \mu)_{\vee(\mathbf{r})}=f(\mathbf{r}) S, \\
s^{\alpha}(\mathbf{r})= & f^{a}(\mathbf{r}) S, \\
s_{k}^{\alpha}= & f_{k}^{\alpha} S .
\end{aligned}
$$

The philicity is defined $\mathrm{as}^{20}$

$$
\omega(\mathbf{r})=\omega f(\mathbf{r}),
$$

and hence

$$
\omega^{\alpha}(\mathbf{r})=\omega f^{\alpha}(\mathbf{r})
$$

and

$$
\omega_{k}^{\alpha}=\omega f_{k}^{\alpha} .
$$

Since these quantities are products of one local and one global reactivity descriptors, they are supposed to explain the intermolecular interactions as well.

Recently Roy and coworkers ${ }^{24,25}$ have tried to check the reliability of the philicity index along with its global counterpart (claimed to be the negative of chemical potential). He has shown ${ }^{24}$ that the philicity properly explains the intermolecular reactivity in most cases for a series of carbonyl compounds although the global electrophilicity fails in many cases. He claims that the relative electrophilicity, originally introduced to avoid negative values of condensed softness ${ }^{26}$ is the most reliable intramolecular reactivity index. His claim is based on the results on the same set of molecules considered in their old paper. ${ }^{27}$ Recently relative electrophilicity has been criticized by several authors ${ }^{28}$ including the manipulation mentioned by Roy himself. ${ }^{29}$ Moreover, counter to the claim of Roy et $a l^{25}$, in the Ref. 11 of his paper ${ }^{24}$ and in many other papers by Contreras, Perez, Fuentealba, Chatterjee, Toro-Labbe and others ${ }^{30-33}$ it has been pointed out that the relative electrophilicity ${ }^{24,27}$ is less universal than the local electrophilicity and also unlike the latter, the former does not provide the proper normalization apart from the fact that it is not trustworthy in interpreting intermolecular interactions. Obviously philicity (like local softness) cannot provide more reliable intramolecular reactivity (when only one molecule is considered) than that is obtainable from the Fukui function but for the cases where the 
molecule is undergoing an intramolecular process like vibration, rotation, rearrangement and/or interaction with a solvent or an external field where both the local and the global descriptors change during the physico-chemical process. The constancy of the external potential is generally tacitly assumed in all such cases. The philicity is, however, not less reliable than the Fukui function and the local softness. In addition philicity and local softness contain information about global electrophilicity and global softness respectively. As shown rather trivially their relative electro(nucleo)philicity ${ }^{24,27}$ is also identical with the corresponding relative Fukui function for the same molecule even for the various intramolecular physico-chemical processes described above and hence does not warrant any additional significance when intramolecular reactivity is analyzed. ${ }^{34}$

His analytical proof based on his (15), does not contain any extra information than the (13) (see also the lines surrounding it) of the original paper of Chattaraj et al. ${ }^{20}$ and his confusion is the result of the improper perusal of that paper (see the point 5 towards the end of ref. 20). The relative electro(nucleo)philicity treats the anions and the cations at par but the anions are known to require a more sophisticated technique than the cations to produce the same quality wavefunctions. The argument ${ }^{25}$ of its being less sensitive to basis set and correlation effects is handwaving. Also the analysis of the radical reactions using these descriptors is not straightforward. Philicity and local softness respectively would be better intermolecular reactivity indices (since a product of a global and a local indices) than the Fukui function to analyze electrophilenucleophile interactions and hard-soft interactions. It may be noted that the numerical results should be taken with care because, as it is well known to the experts in the field of chemical reactivity, the calculation of the Fukui function involves the inherent inadequacies associated with different population analysis schemes/frozen core approximation/finite difference approximation, etc. and the approximations involved in the calculation of the global quantities like relaxation/symmetry/basis set/Koopmans' theorem etc. to provide wrong trends in both intramolecular and intermolecular reactivity studies not necessarily originating from the improper definitions of the various global and local reactivity parameters. Incidentally their claim ${ }^{24,35}$ of guaranty of non-negative Fukui functions from Hirshfeld population analysis scheme has been provided with a counterexample recently. ${ }^{36}$ Moreover, a given reac- tivity descriptor may provide better result in comparison to other for a specific set of molecules and may give worse result for the other set. Incidentally the types of molecules on which the relative electro(nucleo)philicity are calculated so far (see refs $24,25,27,29,35$ and other related references) are very limited and are by no means representative of the wide variety of organic molecules and hence it is premature to claim their descriptor to be the most reliable. ${ }^{24}$ Since philicity and local softness are defined through a decomposition of the corresponding global quantities, local descriptors will definitely reflect the corresponding global reactivity, as opposed to the conclusion drawn by Roy, ${ }^{24}$ if one takes care of all the atomic sites. Of course the major contribution will come from the most reactive site(s). Similar analysis on the connection between local and global properties has been carried out by Legon, ${ }^{37}$ as discussed in ref. [30] in the context of electrophilicity, supporting our argument. During an electrophile-nucleophile interaction process, when two reactants approach each other from a large distance they see each other's global electrophilicities without any idea about their local counterparts. One with the larger electrophilicity will behave as an electrophile and the other as a nucleophile. The most electrophilic site of the electrophile will prefer to interact with the most nucleophilic site of the nucleophile. It may be noted that the atom with the maximum value of the local electrophilicity in the electrophile may not necessarily have larger local electrophilicity value than that of the most electrophilic atom in the nucleophile. A similar situation will arise during hard-soft interaction and will show that the local HSAB principle may not be always in conformity with its global counterpart. If two molecules with different electronegativities (first order effect) approach each other there will be electron transfer between them even if their hardness (second order effect) values are far removed and it is not a violation of the HSAB principle. The Fukui function and all other related descriptors like local softness and philicity may not provide reasonable trends for the hard-hard reactions where charge based descriptors are known to be more appropriate. ${ }^{38}$ Counter to what is claimed by Roy, ${ }^{24}$ philicity has been $\operatorname{shown}^{39}$ to be a reliable descriptor of intramolecular reactivity as well. Meneses et al. ${ }^{39}$ have shown that the philicity model is better than the LiEvans $^{40}$ model and is as reliable as the newly proposed empirical local hardness ${ }^{39}$ in explaining the orientation of the electrophilic aromatic substitution, 
addition reaction, alkylation and acylation of lithium enolates. The reliability of philicity and related descriptors $v i s-\grave{a}-v i s$ that of relative electrophilicity has been analyzed in some more recent papers ${ }^{21,30,32,41,42}$ where the superiority of the former has been highlighted.

In order to avoid misconceptions ${ }^{24,25}$ the relative electro(nucleo)philicity is compared ${ }^{43}$ with philicity for all the molecules considered in references 24 and 25 using same population analysis schemes, same global and local reactivity descriptors, by grouping the molecules as they did, and by analysing in the same line as they did to show that philicity is better than relative electro(nucleo)philicity when the intermolecular reactivity trends are considered and there is hardly any preference of one above the other as far as the intramolecular reactivities are concerned. On the contrary philicity concept has got some advantages over the other concept. ${ }^{43}$

\section{Computational details}

Zeolite is modeled as a trimer cluster (3T) which has been shown ${ }^{1,8,44}$ to be sufficient in representing the Brönsted acid sites of the zeolites and the adsorption of small probe molecules therein. The geometries of the cluster as well as $\mathrm{CH}_{4}, \mathrm{CO}$ and $\mathrm{NH}_{3}$ are optimized using DFT method with Becke, ${ }^{45}$ Lee-YangParr (BLYP) ${ }^{46}$ functional and a double- $\zeta$ numerical polarized (DNP) basis set ${ }^{47}$ from DMol $^{3}$ computer program coupled in the Accelrys package. ${ }^{48}$ The population analysis is performed under the Hirshfeld ${ }^{49}$ scheme.

Using finite difference approximation for the small change in the number of the particles, we can approximate the $\mu, \eta, S$ and $\omega$ as

$$
\begin{aligned}
& \mu=-((I P+E A) / 2), \\
& \eta=(I P-E A) / 2, \\
& S=1 /(I P-E A), \\
& \omega=\mu^{2} / 2 \eta .
\end{aligned}
$$

Equations (6a)-(6c) have been condensed to an atomsin-molecule (AIM) resolution by Yang and Mortier: ${ }^{50}$

$$
\begin{aligned}
& f^{+}=Q_{N^{0}}-Q_{N^{0}+1} \\
& f^{-}=Q_{N^{0}-1}-Q_{N^{0}}
\end{aligned}
$$

$$
f^{0}=\left(Q_{N^{0}-1}-Q_{N^{0}+1}\right) / 2,
$$

where $Q$ is the atomic charge in the molecule. But in our case instead of \pm 1 electron, $\pm 0 \cdot 1$ electron was used by the $\mathrm{DMol}^{3}$ program. ${ }^{48}$ Equations (8c) and (9c) for the local softness and local philicity can be approximated using this scheme and employing (14a)-(14c). Then, we can write

$$
\begin{aligned}
& s^{+}=f^{+} S, \\
& s^{-}=f^{-} S, \\
& s^{0}=f^{0} S,
\end{aligned}
$$

and

$$
\omega^{+}=\omega f^{+},
$$

$$
\omega^{-}=\omega f^{-},
$$

$$
\omega^{0}=\omega f^{0}
$$

For completeness, the supporting information includes results obtained at the Hartree-Fock level using the $6-31 \mathrm{G}^{* *}$ basis set within the Gaussian 98 program. ${ }^{51}$

\section{Results and discussion}

Two different minimum energy structures are obtained for the 3T cluster at BLYP/DNP level of theory as shown in the figures 1 and 2. Pertinent geometrical parameters of these clusters are provided in table 1. Bond lengths and bond angles of one of them compare very well with those reported by Deka et al. ${ }^{8}$

Table 2 contains the global reactivity parameters; chemical potential, global softness and global electrophilicity for $3 \mathrm{~T}$ clusters, $\mathrm{CH}_{4}, \mathrm{CO}$ and $\mathrm{NH}_{3}$. The strongest interaction is between the zeolite and $\mathrm{NH}_{3}$ according to the HSAB principle since they are the two softest species considered here as per their global softness values. It is important to note that the zeolite is a better electrophile and $\mathrm{NH}_{3}$ is a better nucleophile (poorer electrophile) and hence will have strong interaction. Since CO is a better electrophile than the zeolite its nucleophilic character may not be properly ascertained during its reaction with the zeolite as hinted by others. ${ }^{5}$ 
Table 1. Pertinent geometrical parameters of $3 \mathrm{~T}$ cluster calculated using DFT method at BLYP/DNP level of theory.

\begin{tabular}{lccc}
\hline Parameter & 3T cluster-1(*) & 3T cluster2 $(*)$ & 3T $(* *)$ \\
\hline $\begin{array}{l}\text { Bond lengths }(\AA) \\
\mathrm{O}_{4}-\mathrm{H}_{8}\end{array}$ & 0.975 & 0.973 & \\
$\mathrm{Si}_{-} \mathrm{O}_{4}$ & 1.740 & 1.746 & 0.973 \\
$\mathrm{Al}-\mathrm{O}_{4}$ & 1.990 & 1.979 & 1.745 \\
$\mathrm{Al}-\mathrm{O}_{3}$ & 1.738 & 1.739 & 1.976 \\
$\mathrm{Al}-\mathrm{O}_{5}$ & 1.750 & 1.756 & 1.739 \\
$\mathrm{Al}-\mathrm{O}_{6}$ & 1.744 & 1.738 & 1.738 \\
Bond angles (deg) & & & \\
$\mathrm{Si}-\mathrm{O}_{4}-\mathrm{Al}$ & $122 \cdot 7$ & 121.2 & 122 \\
$\mathrm{Si}-\mathrm{O}_{4}-\mathrm{H}_{8}$ & $116 \cdot 8$ & 117.3 & 117 \\
$\mathrm{Al}-\mathrm{O}_{4}-\mathrm{H}_{8}$ & 113.8 & 121.2 & 121 \\
\hline
\end{tabular}

*Present work; **From Deka et al ${ }^{8}$

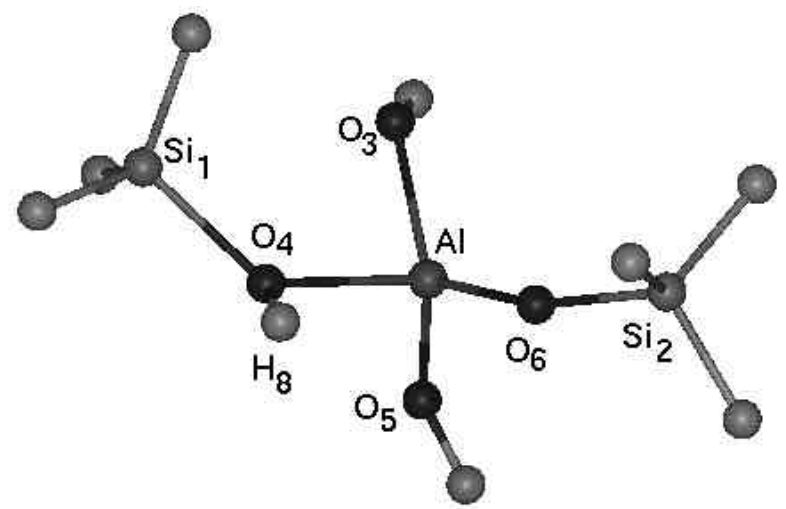

Figure 1. 3T H-ZSM-5 cluster model (3T cluster-1) used to simulate the active site of the H-ZSM-5 zeolite. It contains three tetrahedral units.

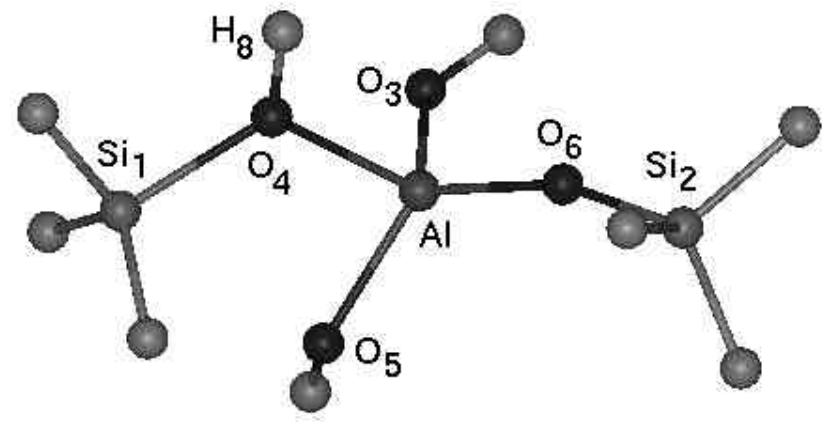

Figure 2. 3T cluster model (3T cluster-2) used to simulate the active site of the zeolite. It contains three tetrahedral units. Bond lengths and bond angles compare well with that reported by Deka et al. ${ }^{8}$

Various local quantities like the Fukui function, local softness and local philicity are presented in table 3 . Apart from the silicon-1 site, the acidic hydrogen $\left(\mathrm{H}_{8}\right)$ site in the zeolite is the most reactive, the soft-
Table 2. Global reactivity descriptors for the zeolite cluster and molecules (in atomic units), using finite difference approximation for the small change in the number of particles ( $\pm 0 \cdot 1$ electron), at BLYP/DNP level of theory.

\begin{tabular}{lccc}
\hline System & $\begin{array}{c}\text { Chemical } \\
\text { potential }\end{array}$ & $\begin{array}{c}\text { Global } \\
\text { softness }\end{array}$ & $\begin{array}{c}\text { Global } \\
\text { electrophilicity }\end{array}$ \\
\hline 3T cluster-1 & $-0 \cdot 14759$ & 4.99226 & $0 \cdot 10874$ \\
3T cluster-2 & $-0 \cdot 14855$ & 4.96396 & $0 \cdot 10954$ \\
$\mathrm{CH}_{4}$ & $-0 \cdot 15472$ & $2 \cdot 43032$ & $0 \cdot 05818$ \\
$\mathrm{CO}$ & $-0 \cdot 19570$ & $3 \cdot 30739$ & $0 \cdot 12667$ \\
$\mathrm{NH}_{3}$ & $-0 \cdot 11594$ & $3 \cdot 89747$ & $0 \cdot 05239$ \\
\hline
\end{tabular}

est and the most apt for nucleophilic attack. The philicity values of $\mathrm{N}$ in $\mathrm{NH}_{3}$ and of $\mathrm{C}$ in $\mathrm{CH}_{4}$ and $\mathrm{CO}$ are the largest and these sites are preferable for adsorption into the $\mathrm{H}_{8}$-site of the zeolite, through favorable electrophile-nucleophile interactions, complementing a local HSAB result ${ }^{22}$ reported by Deka $e l$ $a l^{8}$ who have shown through the calculations of interaction energy that the local HSAB principle should be realized via a softness- matching criterion rather than a Fukui function matching criterion as the confusion was there before. ${ }^{5}$ It is important to note that the Fukui function and local softness provide different trends ${ }^{8}$ as will be the case with philicity. ${ }^{20}$ Respective criteria are based on three different chemical theories of reactivity and selectivity, viz., preferred interactions are through maximum Fukui function sites (frontier electron theory), hard-hard or soft-soft sites (local HSAB principle) and maximum electrophilic site of the electrophile-maximum nucleophilic site of the nucleophile (best electrophilenucleophile interactions). Philicity based study is somewhat better in the sense that it can account for most of the cases studied here as opposed to several 
Table 3. Condensed Fukui function, local softness and local philicity values for the zeolite cluster and molecules (in atomic units). The quantities are calculated by means of a finite-difference approximation ( $\pm 0 \cdot 1$ electron) calculated using DFT method (BLYP/DNP) and Hirshfeld population scheme.

\begin{tabular}{|c|c|c|c|}
\hline System & Fukui function & Local softness & Local philicity \\
\hline $3 T$ cluster -1 & $f^{+}$ & $s^{+}$ & $w^{+}$ \\
\hline $\mathrm{H}_{8}$ & $0 \cdot 14500$ & 0.72388 & $0 \cdot 01577$ \\
\hline $\mathrm{Al}$ & $0 \cdot 06800$ & $0 \cdot 33947$ & 0.00739 \\
\hline $\mathrm{O}_{3}$ & $0 \cdot 01400$ & 0.06989 & $0 \cdot 00152$ \\
\hline $\mathrm{O}_{4}$ & 0.07700 & $0 \cdot 38440$ & 0.00837 \\
\hline $\mathrm{O}_{5}$ & $0 \cdot 02400$ & $0 \cdot 11981$ & $0 \cdot 00261$ \\
\hline $\mathrm{O}_{6}$ & $0 \cdot 00400$ & 0.01997 & $0 \cdot 00043$ \\
\hline $\mathrm{Si}_{1}$ & $0 \cdot 30300$ & $1 \cdot 51265$ & 0.03295 \\
\hline $\mathrm{Si}_{2}$ & $0 \cdot 04100$ & $0 \cdot 20468$ & $0 \cdot 00446$ \\
\hline $3 T$ cluster-2 & $f^{+}$ & $s^{+}$ & $w^{+}$ \\
\hline $\mathrm{H}_{8}$ & $0 \cdot 16900$ & 0.83891 & $0 \cdot 01851$ \\
\hline $\mathrm{Al}$ & $0 \cdot 07800$ & $0 \cdot 38719$ & $0 \cdot 00854$ \\
\hline $\mathrm{O}_{3}$ & $0 \cdot 02400$ & $0 \cdot 11914$ & $0 \cdot 00263$ \\
\hline $\mathrm{O}_{4}$ & 0.07700 & $0 \cdot 38222$ & $0 \cdot 00843$ \\
\hline $\mathrm{O}_{5}$ & 0.02000 & 0.09928 & 0.00219 \\
\hline $\mathrm{O}_{6}$ & $0 \cdot 00400$ & 0.01986 & $0 \cdot 00044$ \\
\hline $\mathrm{Si}_{1}$ & $0 \cdot 27500$ & $1 \cdot 36509$ & 0.03012 \\
\hline $\mathrm{Si}_{2}$ & $0 \cdot 03700$ & $0 \cdot 18367$ & 0.00405 \\
\hline $\mathrm{CH}_{4}$ & $f^{-}$ & $s^{-}$ & $w^{-}$ \\
\hline $\mathrm{C}$ & $0 \cdot 30800$ & 0.74854 & $0 \cdot 01792$ \\
\hline $\mathrm{H}$ & $0 \cdot 17300$ & $0 \cdot 42045$ & $0 \cdot 01007$ \\
\hline $\mathrm{CO}$ & $f^{-}$ & $s^{-}$ & $w^{-}$ \\
\hline $\mathrm{C}$ & 0.69700 & $2 \cdot 30525$ & 0.08829 \\
\hline $\mathrm{O}$ & $0 \cdot 30300$ & $1 \cdot 00214$ & 0.03838 \\
\hline $\mathrm{NH}_{3}$ & $f^{-}$ & $s^{-}$ & $w^{-}$ \\
\hline $\mathrm{N}$ & $0 \cdot 52500$ & $2 \cdot 04617$ & $0 \cdot 02750$ \\
\hline $\mathrm{H}$ & $0 \cdot 15800$ & $0 \cdot 61580$ & $0 \cdot 00828$ \\
\hline
\end{tabular}

violations in the proper softness matching (on local HSAB) criterion as seen in the present work as well as that of Deka et al. ${ }^{8}$ Philicity results are in conformity with frontier orbital theory since the interactions between sites of maximum Fukui function values (hence the most reactive) are most favorable according to their philicity values also although with different $\omega$ values, but counter to that claimed by Deka et $a l^{8}$ regarding the Fukui function. Similar analysis on adsorption of $\mathrm{CO}, \mathrm{NH}_{3}$ and $\mathrm{H}_{2} \mathrm{O}$ in faujasite $x$-type zeolite was made by Krishnamurty $e t a l^{5}$ using local HSAB principle which provided similar quantitative results on a qualitative basis. Before the related work by Deka $e t a l^{8}$ a quantitative extension of that study ${ }^{5}$ to calculate interaction energies between zeolite and $\mathrm{N}_{2}, \mathrm{CO}_{2}$ and $\mathrm{CO}$ was also reported ${ }^{6}$. These results are interesting although the minimization of a local grand potential ${ }^{22}$ and use of an adjustable parameter made this analysis weak ${ }^{52}$.
The quantities calculated with the HF/6-31G**// BLYP/DNP procedure by using both finite-difference and Koopmans' approximations with Mulliken as well as Hirshfeld charges are provided as supporting information. In most of the cases the active sites of the zeolite $\left(\mathrm{H}_{8}\right), \mathrm{CH}_{4}(\mathrm{C}), \mathrm{CO}(\mathrm{C})$ and $\mathrm{NH}_{3}(\mathrm{~N})$ are properly accounted for. In the present work, mainly Hirshfeld population is used because Mulliken population provides negative values for the local quantities like the Fukui function, local softness and local philicity in many cases as in ref. [8].

\section{Concluding remarks}

Different global and local density functional theory based descriptors are calculated for understanding the nature of interaction, reactivity and selectivity associated with the adsorption of small molecules such as $\mathrm{CH}_{4}, \mathrm{CO}$ and $\mathrm{NH}_{3}$ to the Brönsted acidic 
sites of zeolites. Interaction between the zeolite and $\mathrm{NH}_{3}$ is the strongest because it results from the combination of two softest species as well as between the best electrophile and the nucleophile. The $\mathrm{N}$ atom in $\mathrm{NH}_{3}$ and the $\mathrm{C}$ atoms in $\mathrm{CH}_{4}$ and $\mathrm{CO}$ are most active towards adsorption to the acid sites of the zeolite. Philicity concept, frontier orbital theory and local HSAB principle provide identical inferences based on local philicities, Fukui functions and local softnesses.

\section{Acknowledgements}

Authors thank the Mexican Institute of Petroleum for facilities, MG thanks CONACYT-México for financial support and A C acknowledges the Mexican Institute of Petroleum and CONACYT-México for the $\mathrm{Ph} \mathrm{D}$ scholarship. We would like to thank the reviewers for very constructive criticisms especially for suggesting that we compare the relative merits and demerits of philicity in comparison to those of relative electrophilicity. Thanks are also due to $\mathrm{Mr} \mathrm{U}$ Sarkar and Mr D R Roy for their help with the manuscript.

\section{References}

1. (a) van Santen R A and Kramer G J 1995 Chem. Rev. 95 637; (b) Kramer G J, van Santen R A, Emeis C A and Nowak A K 1993 Nature (London) 363529

2. Sauer J, Ugliengo P, Garrone E and Saunders V R 1994 Chem. Rev. 942095

3. Vetrivel R and Catlow C R A 1992 In Modelling of structure and reactivity of zeolites (ed.) C R A Catlow (London: Academic Press) p. 217

4. Stave M S and Nicholas J B 1995 J. Phys. Chem. 99 15046; Bates S P and Dwyer J 1993 J. Phys. Chem. 97 5897; Langenaeker W, Goussement N, De Proft F and Geerlings P 1994 J. Phys. Chem. 983010

5. Krishnamurti S M, Roy R K, Vetrivel R, Iwata S and Pal S 1997 J. Phys. Chem. 1017253

6. Pal S and Chandrakumar K R S $2000 \mathrm{~J}$. Am. Chem. Soc. 1224145

7. Deka R C, Vetrivel R and Pal S 1999 J. Phys. Chem. A103 5978

8. Deka R C, Ajitha D and Hirao K 2003 J. Phys. Chem. B107 8574

9. Parr R G and Yang W 1989 Density functional theory of atoms and molecules (New York: Oxford University Press)

10. Geerlings P, De Proft F and Langenaeker W 2003 Chem. Rev. 1031793

11. Parr R G, Donnelly D A, Levy M and Palke W E 1978 J. Chem. Phys. 683801

12. Sen K D and Jorgenson C K 1987 Electronegativity: Structure and bonding (Berlin: Springer-Verlag) vol 66
13. Parr R G and Pearson R G 1983 J. Am. Chem. Soc. 1057512

14. Parr R G and Mingos D M P 1993 Chemical hardness: Structure and bonding (Berlin: Springer) vol 80

15. Pearson R G 1997 Chemical hardness: Application from molecules to solid (Weinheim: Wiley-VCH)

16. (a) Parr R G, Szentpály L and Liu S 1999 J. Am. Chem. Soc. 121 1922; (b) Maynard A T, Huang M, Rice W G and Covell D G 1998 Proc. Natl. Acad. Sci. USA 9511578

17. Parr R G and Yang W 1984 J. Am. Chem. Soc. 106 1976

18. Ayers P W and Levy M 2000 Theor. Chem. Acc. 103 353

19. Yang W and Parr R G 1985 Proc. Natl. Acad. Sci. USA 821960

20. Chattaraj P K, Maiti B and Sarkar U 2003 J. Phys. Chem. A107 4973

21. Chattaraj P K, Lee H and Parr R G 1991 J. Am. Chem. Soc. 1131855

22. Gazquez J L and Mendez F 1994 J. Phys. Chem. 98 4591, and references therein

23. Fukui K 1982 Science 218747

24. Roy R K 2004 J. Phys. Chem. A108 4934

25. Roy R K, Usha V, Paulovi J and Hirao K $2005 \mathrm{~J}$. Phys. Chem. A109 4601

26. Page 1812: Geerlings P, De Proft F and Langenaeker W 2003 Chem. Rev. 1031793

27. Roy R K, Krishnamurti S, Geerlings P and Pal S 1998 J. Phys. Chem. A102 3746

28. Olah J, van Alsenoy C and Sannigrahi A B $2002 J$. Phys. Chem. A106 3885; Tishchenko O, Pham-Tran N, Kryachko E S and Nguyen T M 2001 J. Phys. Chem. A105 8709; Fuentealba P and Contreras R R In Reviews of modern quantum chemistry (ed.) K D Sen (Singapore: World Scientific) pp 1013-1052

29. Roy R K 2003 J. Phys. Chem. A107 397; Roy R K 2003 J. Phys. Chem. A107 10428

30. Pérez P, Toro-Labbé A, Aizman A and Contreras R 2002 J. Org. Chem. 674747 ; Domingo L R, Aurell M J, Pérez P and Contreras R 2003 J. Org. Chem. 68 3884 ; Domingo L R, Aurell M J, Pérez P and Contreras R 2002 J. Phys. Chem. A106 6871 ; In the abstract of the last paper, the major problems associated with relative electrophilicity over local electrophilicity are highlighted

31. Meneses L, Tiznado W, Contreras R and Fuentealba P 2004 Chem. Phys. Lett. 383 181. See also, Domingo L R, Pérez P and Contreras R 2004 Tetrahedron 60 6585, where it has been argued that the local electrophilicity can help elucidate the intramolecular selectivity properly

32. Morrell C, Grand A and Toro-Labbe A 2005 J. Phys. Chem. A109 205

33. Chatterjee A, Ebina T and Iwasaki T 2001 J. Phys. Chem. A105 10694

34. Chattaraj P K and Sarkar U 2003 Proceedings of IECMD (This paper was withdrawn due to delay in publication). Portions of this work were presented in TTC 2002 at IACS, Kolkata and TCS 2004 in BARC, Mumbai 
35. Roy R K, Pal S and Hirao K 1999 J. Chem. Phys. 110 8236; Roy R K, Hirao K and Pal S $2000 \mathrm{~J}$. Chem. Phys. 113 1372; Roy R K, Tajima N and Hirao K 2001 J. Phys. Chem. A105 2117; Roy R K 2003 J. Phys. Chem. A107 397; Roy R K 2003 J. Phys. Chem. A107 10428

36. Mañanes A, Duque F, Méndez F, López M J and Alonso J A 2003 J. Chem., Phys. 1195128

37. Legon A C 1999 Angew. Chem., Int. Ed. 38 2686; Legon A C and Millen D J 1987 J. Am. Chem. Soc. 109356

38. Chattaraj P K 2001 J. Phys. Chem. A105 511; Melin J, Aparicio F, Subramanian V, Galvan M and Chattaraj P K 2004 J. Phys. Chem. A108 2487; Hocquet A, Toro-Labbé A and Chermette H 2004 J. Mol. Struct. (Theochem.) 686213

39. Meneses L, Tiznado W, Contreras R and Fuentealba P 2004 Chem. Phys. Lett. 383181

40. Li Y and Evans J N S 1995 J. Am. Chem. Soc. 117 7756

41. Chattaraj P K, Sarkar U, Parthasarathi R and Subramanian V 2005 Int. J. Quantum Chem. 101690
42. Parthasarathi R, Padmanabhan J, Elango M, Subramanian V and Chattaraj P K 2004 Chem. Phys. Lett. 394225

43. Elango M, Parthasarathi R, Subramanian V and Chattaraj P K Int. J. Quantum Chem. (in press); Roy D R, Parthasarathi R, Padmanabhan J, Sarkar U, Subramanian V and Chattaraj P K (unpublished work)

44. Deka R C, Roy R K and Hirao K 2000 Chem. Phys. Lett. 332576

45. Becke A D 1988 J. Chem. Phys. 882547

46. Lee C, Yang W and Parr R G 1988 Phys. Rev. B37 786

47. Delley B and Ellis D E 1982 J. Chem. Phys. 761949

48. $\mathrm{DMol}^{3}$ Module of Cerius2 by Accelrys Corp. San Diego, CA

49. Hirshfeld F L 1977 Theor. Chim. Acta B44 129

50. Yang W and Mortier W J 1986 J. Am. Chem. Soc. 1085708

51. Frisch M et al 1998 Gaussian 98 A.7, Gaussian, Inc, Pittsburgh, PA

52. Chattaraj P K 2001 J. Phys. Chem. A105 511; Ayers P W 2005 J. Chem. Phys. 122141102

Supporting information (Tables S4-S6) is available on the Journal website: www.ias.ac.in/chemsci 